\title{
Regularity for weakly Dirac-harmonic maps to hypersurfaces
}

\author{
Miaomiao Zhu
}

Received: 25 March 2008 / Accepted: 7 October 2008 / Published online: 13 November 2008

(C) Springer Science+Business Media B.V. 2008

\begin{abstract}
We prove that a weakly Dirac-harmonic map from a Riemann spin surface to a compact hypersurface $N \subset \mathbb{R}^{d+1}$ is smooth.
\end{abstract}

Keywords Dirac-harmonic map $\cdot$ Regularity $\cdot$ Hypersurface

Mathematics Subject Classification (2000) 58J05 · 53C27

\section{Introduction}

As critical points of an action functional, which is motivated by the supersymmetric nonlinear sigma model from quantum field theory [3], Dirac-harmonic maps are defined as solutions of a system of nonlinear elliptic equations which couples harmonic-type equations and Dirac-type equations in a natural way [2].

It is observed in [2] that the conformal invariance of harmonic maps in two dimensions and Dirac operators in arbitrary finite dimensions are preserved in the case of two-dimensional Dirac-harmonic maps. This brings the study of Dirac-harmonic maps into the framework of two-dimensional conformally invariant variational problems. As a typical problem in calculus of variation, the regularity issue for weak solutions is of particular importance. For the regularity of weakly harmonic maps, see for instance [5,6].

Chen et al. [1] studied the regularity problem for Dirac-harmonic map and proved that any weakly Dirac-harmonic map from a spin surface to the standard sphere $\mathbb{S}^{d}$ is actually smooth. They [1] observed a Jacobian structure of the Dirac-harmonic map equation for spherical target manifold and proved the regularity property by applying Wente's lemma [11]. However, when the target are general compact Riemannian manifolds, the spinor terms

Supported by IMPRS "Mathematics in the Sciences" and the Klaus Tschira Foundation.

M. Zhu $(\bowtie)$

Max Planck Institute for Mathematics in the Sciences, Inselstr.22-26, 04103 Leipzig, Germany

e-mail: Miaomiao.Zhu@ mis.mpg.de 
in the harmonic-type equations for Dirac-harmonic maps cannot be written as a Jacobian form and thus Wente's lemma cannot be applied to obtain the regularity.

Recently, Rivière [10] discovered the existence of a conservation law for weak solutions of the Euler-Lagrange equations of conformally invariant variational integrals in two dimensions. He succeeded in writing two-dimensional conformally invariant nonlinear elliptic PDEs (e.g. harmonic map equations, prescribed mean curvature equations, etc.) in divergence form, from which one can recover all the classical regularity results for such weak solutions and obtain new results.

In this paper, we observe that, when the target is a compact hypersurface $N \subset \mathbb{R}^{d+1}$, the harmonic-type equations for Dirac-harmonic maps can be written in a special form to which Rivière's regularity results can be applied. Our result is the following:

Theorem 1.1 A weakly Dirac-harmonic map $(\phi, \psi)$ from a Riemann spin surface $M$ to a compact hypersurface $N \subset \mathbb{R}^{d+1}$ is smooth.

\section{Dirac-harmonic maps}

Let $(M, h)$ be an oriented, compact Riemannian surface with a fixed spin structure. Let $\Sigma M$ be the spinor bundle over $M$ with a hermitian metric $\langle\cdot, \cdot\rangle_{\Sigma M}$ and a compatible spin connection $\nabla$. For $X \in \Gamma(T M), \psi \in \Gamma(\Sigma M)$, we denote by $X \cdot \psi$ the Clifford multiplication, which satisfies the following relations:

$$
\langle X \cdot \psi, \varphi\rangle=-\langle\psi, X \cdot \varphi\rangle, \quad X \cdot Y \cdot \psi+Y \cdot X \cdot \psi=-2 h(X, Y) \psi,
$$

for $X, Y \in \Gamma(T M), \psi, \varphi \in \Gamma(\Sigma M)$. The Dirac operator $\not \partial$ is defined by $\not \partial \psi:=e_{\alpha} \cdot \nabla_{e_{\alpha}} \psi$ for a local orthonormal frame $\left\{e_{1}, e_{2}\right\}$ of $T M$ and $\psi \in \Gamma(\Sigma M)$. The summation convention will be used throughout the paper. We refer to $[4,7,9]$ for more spin geometric materials.

Let $(N, g)$ be a compact Riemanian manifold of dimension $d \geq 2$, and let its metric in local coordinates be given by $g_{i j}$, with Christoffel symbols $\Gamma_{j k}^{i}$ and Riemannian curvature tensor $R_{l i j}^{m}$. Let $\phi$ be a smooth map from $M$ to $N$ and $\phi^{*} T N$ the pull-back bundle of $T N$ under $\phi$. Consider the twisted bundle $\Sigma M \otimes \phi^{*} T N$ with the induced metric $\langle\cdot, \cdot\rangle_{\Sigma M \otimes \phi^{*} T N}$ and the induced connection $\widetilde{\nabla}:=\nabla \otimes 1+1 \otimes \nabla^{\phi^{*} T N}$. We write the section $\psi$ of $\Sigma M \otimes \phi^{*} T N$ locally as follows:

$$
\psi(x)=\psi^{i}(x) \otimes \partial_{y^{i}}(\phi(x))
$$

where $\psi^{i}$ is a spinor and $\left\{\partial_{y^{i}}\right\}$ is a local basis on $N$. Then, $\widetilde{\nabla}$ can be expressed by

$$
\widetilde{\nabla} \psi=\nabla \psi^{i}(x) \otimes \partial_{y^{i}}(\phi(x))+\Gamma_{j k}^{i} d \phi^{j}(x) \psi^{k}(x) \otimes \partial_{y^{i}}(\phi(x)) .
$$

The Dirac operator along the map $\phi$ is defined by

$$
\begin{aligned}
\not D \psi & :=e_{\alpha} \cdot \widetilde{\nabla}_{e_{\alpha}} \psi \\
& =\not \partial \psi^{i}(x) \otimes \partial_{y^{i}}(\phi(x))+\Gamma_{j k}^{i} \nabla_{e_{\alpha}} \phi^{j}(x) e_{\alpha} \cdot \psi^{k}(x) \otimes \partial_{y^{i}}(\phi(x)) .
\end{aligned}
$$

Set

$$
\chi(M, N):=\left\{(\phi, \psi) \mid \phi \in C^{\infty}(M, N), \psi \in \Gamma\left(\Sigma M \otimes \phi^{*} T N\right)\right\}
$$


We consider the following functional defined on $\chi(M, N)$ :

$$
\begin{aligned}
L(\phi, \psi) & :=\int_{M}\left(|d \phi|^{2}+\langle\psi, \not D \psi\rangle\right) \\
& =\int_{M}\left(g_{i j}(\phi) h^{\alpha \beta} \frac{\partial \phi^{i}}{\partial x_{\alpha}} \frac{\partial \phi^{j}}{\partial x_{\beta}}+g_{i j}(\phi)\left\langle\psi^{i}, \not D \psi^{j}\right\rangle_{\Sigma M}\right) \sqrt{\operatorname{det}\left(\mathrm{h}_{\alpha \beta}\right)} d x^{1} d x^{2},
\end{aligned}
$$

where in the second formula the integral is expressed in terms of local data. The corresponding Euler-Lagrange equations are

$$
\begin{gathered}
\Delta \phi^{m}+\Gamma_{j k}^{m}(\phi) h^{\alpha \beta} \partial_{\alpha} \phi^{j} \partial_{\beta} \phi^{k}=\frac{1}{2} R_{l i j}^{m}(\phi)\left\langle\psi^{i}, \nabla \phi^{l} \cdot \psi^{j}\right\rangle, \\
\not \psi^{i}+\Gamma_{j k}^{i}(\phi) \partial_{\alpha} \phi^{j} e_{\alpha} \cdot \psi^{k}=0 .
\end{gathered}
$$

Here, $\nabla \phi^{l}:=\partial_{\alpha} \phi^{l} e_{\alpha}=\phi_{\alpha}^{l} e_{\alpha}$ and “." denotes the Clifford multiplication. Set

$$
\mathcal{R}(\phi, \psi)(x):=\frac{1}{2} R_{l i j}^{m}(\phi(x))\left\langle\psi^{i}, \nabla \phi^{l} \cdot \psi^{j}\right\rangle \partial_{y^{m}}(\phi(x)) .
$$

Then, (1) and (2) can be written in the following global form

$$
\begin{gathered}
\tau(\phi)=\mathcal{R}(\phi, \psi), \\
\not D \psi=0,
\end{gathered}
$$

where $\tau(\phi) \in \Gamma\left(\phi^{*} T N\right)$ is the tension field of the map $\phi$. Solutions $(\phi, \psi)$ of (1), (2) are called Dirac-harmonic maps from $M$ to $N$.

Note that (1) and (2) are the intrinsic versions of the Dirac-harmonic map equations. Now we shall give the extrinsic version of these equations. By the Nash-Moser embedding theorem, we embed $N$ into some $\mathbb{R}^{K}$. Let $A$ be the second fundamental form of $N$ in $\mathbb{R}^{K}$ and $P$ the shape operator. It is not difficult to verify the following relation:

$$
\langle P(\xi ; X), Y\rangle_{T N}=\langle A(X, Y), \xi\rangle_{\mathbb{R}^{K}}
$$

for any $X, Y \in \Gamma(T N), \xi \in \Gamma\left(T^{\perp} N\right)$, where $T^{\perp} N$ is the normal bundle to $N$. Then, by the Gauss equation (see [8]), we have

$$
\begin{aligned}
R_{l i j}^{m}\left\langle\psi^{i}, \nabla \phi^{l} \cdot \psi^{j}\right\rangle= & g^{m k}\left\langle A\left(\partial_{y^{k}}, \partial_{y^{i}}\right), A\left(\partial_{y^{l}}, \partial_{y^{j}}\right)\right\rangle\left\langle\psi^{i}, \nabla \phi^{l} \cdot \psi^{j}\right\rangle \\
& \left.-\left\langle A\left(\partial_{y^{k}}, \partial_{y^{j}}\right), A\left(\partial_{y^{l}}, \partial_{y^{i}}\right)\right\rangle\right)\left\langle\psi^{i}, \nabla \phi^{l} \cdot \psi^{j}\right\rangle \\
= & g^{m k}\left\langle A\left(\partial_{y^{k}}, \partial_{y^{i}}\right), A\left(\partial_{y^{l}}, \partial_{y^{j}}\right)\right\rangle\left\langle\psi^{i}, \nabla \phi^{l} \cdot \psi^{j}\right\rangle \\
& +g^{m k}\left\langle A\left(\partial_{y^{k}}, \partial_{y^{i}}\right), A\left(\partial_{y^{l}}, \partial_{y^{j}}\right)\right\rangle\left\langle\psi^{i}, \nabla \phi^{l} \cdot \psi^{j}\right\rangle \\
= & 2 \operatorname{Re} g^{m k}\left\langle A\left(\partial_{y^{k}}, \partial_{y^{i}}\right), A\left(\partial_{y^{l}}, \partial_{y^{j}}\right)\right\rangle\left\langle\psi^{i}, \nabla \phi^{l} \cdot \psi^{j}\right\rangle \\
= & 2 \operatorname{Re} g^{m k}\left\langle P\left(A\left(\partial_{y^{l}}, \partial_{y^{j}}\right) ; A\left(\partial_{y^{i}}\right), \partial_{y^{k}}\right)\left\langle\psi^{i}, e_{\alpha} \cdot \psi^{j}\right\rangle \phi_{\alpha}^{l},\right.
\end{aligned}
$$

where in the last step we used (5). Set

$$
\begin{aligned}
\mathcal{A}\left(d \phi\left(e_{\alpha}\right), e_{\alpha} \cdot \psi\right) & :=\phi_{\alpha}^{i} e_{\alpha} \cdot \psi^{j} \otimes A\left(\partial_{y^{i}}, \partial_{y^{j}}\right), \\
\mathcal{P}\left(\mathcal{A}\left(d \phi\left(e_{\alpha}\right), e_{\alpha} \cdot \psi\right) ; \psi\right) & :=P\left(A\left(\partial_{y^{l}}, \partial_{y^{j}}\right) ; \partial_{y^{i}}\right)\left\langle\psi^{i}, e_{\alpha} \cdot \psi^{j}\right\rangle \phi_{\alpha}^{l} .
\end{aligned}
$$


Then, Equs. (1) and (2) become the following form:

$$
\begin{gathered}
-\Delta \phi=A\left(d \phi\left(e_{\alpha}\right), d \phi\left(e_{\alpha}\right)\right)+\operatorname{Re} \mathcal{P}\left(\mathcal{A}\left(d \phi\left(e_{\alpha}\right), e_{\alpha} \cdot \psi\right) ; \psi\right), \\
\not \psi=\mathcal{A}\left(d \phi\left(e_{\alpha}\right), e_{\alpha} \cdot \psi\right) .
\end{gathered}
$$

Here, $\phi$ is a map from $M$ to $\mathbb{R}^{K}$ with

$$
\phi(x) \in N
$$

for any $x \in M$, and the spinor field $\psi$ along the map $\phi$ is a $K$-tuple of spinors $\left(\psi^{1}, \psi^{2}, \ldots, \psi^{K}\right)$ satisfying

$$
\sum_{i} v^{i} \psi^{i}=0, \text { for any normal vector } v=\sum_{i=1}^{K} v^{i} E_{i} \text { at } \phi(x),
$$

where $\left\{E_{i}, i=1,2, \ldots, K\right\}$ is the standard basis of $\mathbb{R}^{K}$. Set

$$
\chi_{1,4 / 3}^{1,2}(M, N):=\left\{(\phi, \psi) \in W^{1,2} \times W^{1,4 / 3} \text { with (11) and (12) a.e. }\right\} .
$$

Then the functional $L(\phi, \psi)$ is well defined for $(\phi, \psi) \in \chi_{1,4 / 3}^{1,2}(M, N)$. Critical points $(\phi, \psi)$ of the functional $L(\phi, \psi)$ in $\chi_{1,4 / 3}^{1,2}(M, N)$ are called weakly Dirac-harmonic maps from $M$ to $N$ (see [1]).

Note that $\mathcal{A}, \mathcal{P}$ in (7), (8) are defined in terms of a fixed local coordinates of $N$. This requires the continuity of the map $\phi$. For weakly Dirac-harmonic maps, we need an extrinsic version of the equations, where everything is written down in coordinates of the ambient space $\mathbb{R}^{K}$. To this end, we fix the canonical coordinates $\left(y^{1}, y^{2}, \ldots, y^{K}\right)$ of $\mathbb{R}^{K}$. Let $v_{l}$, $l=d+1, \ldots, K$ be an orthonomal frame field for the normal bundle $T^{\perp} N$ to $N$. We denote the corresponding section of $\phi^{*}\left(T^{\perp} N\right)$ with the same symbol $v_{l}$. Then, for $X=X^{i} \partial_{y^{i}} \in$ $T_{y} N, Y=Y^{j} \partial_{y^{j}} \in T_{y} N, Z=Z^{l} \partial_{y^{l}} \in T_{y} N$, we have $\nabla_{Y} v_{k}=\sum_{i=1}^{K} Y^{i} \frac{\partial v_{k}}{\partial y^{i}}$, and the following holds (c.f. [6], Chap. 1):

$$
\begin{gathered}
A(y)(X, Y)=\left\langle X, \nabla_{Y} v_{l}\right\rangle v_{l}=\left\langle X, Y^{j} \frac{\partial v_{l}}{\partial y^{j}}\right\rangle v_{l}=X^{i} Y^{j} \frac{\partial v_{l}^{i}}{\partial y^{j}} v_{l}, \\
P(A(X, Y) ; Z)=\left(\nabla_{Z} v_{l}\right)^{\top} X^{i} Y^{j} \frac{\partial v_{l}^{i}}{\partial y^{j}}=\left(\frac{\partial v_{l}}{\partial y^{k}}\right)^{\top} X^{i} Y^{j} Z^{k} \frac{\partial v_{l}^{i}}{\partial y^{j}},
\end{gathered}
$$

where $\top$ denotes the projection $: \mathbb{R}^{K} \rightarrow T_{y} N$. In view of (7), (8), we can write $\mathcal{A}, \mathcal{P}$ as follows, for $X=X^{i} \partial_{y^{i}} \in T_{y} N, \psi=\psi^{j} \partial_{y^{j}} \in \Sigma M \otimes T_{y} N, \varphi=\varphi^{k} \partial_{y^{k}} \in \Sigma M \otimes T_{y} N$,

$$
\begin{gathered}
\mathcal{A}(X, \psi)=X^{i} \psi^{j} \otimes \frac{\partial v_{l}^{i}}{\partial y^{j}} v_{l} \\
\mathcal{P}(\mathcal{A}(X, \psi) ; \varphi)=\left(\frac{\partial v_{l}}{\partial y^{k}}\right)^{\top} X^{i}\left\langle\varphi^{k}, \psi^{j}\right\rangle \frac{\partial v_{l}^{i}}{\partial y^{j}} .
\end{gathered}
$$

By the symmetry of $A(X, Y)$ with respect to $X$ and $Y$, we have

$$
\begin{gathered}
X^{i} \psi^{j} \otimes \frac{\partial v_{l}^{i}}{\partial y^{j}} v_{l}=X^{j} \psi^{i} \otimes \frac{\partial v_{l}^{i}}{\partial y^{j}} v_{l} \\
\left(\frac{\partial v_{l}}{\partial y^{k}}\right)^{\top} X^{i}\left\langle\varphi^{k}, \psi^{j}\right\rangle \frac{\partial v_{l}^{i}}{\partial y^{j}}=\left(\frac{\partial v_{l}}{\partial y^{k}}\right)^{\top} X^{j}\left\langle\varphi^{k}, \psi^{i}\right\rangle \frac{\partial v_{l}^{i}}{\partial y^{j}} .
\end{gathered}
$$


Consider a weakly Dirac-harmonic map $(\phi, \psi) \in \chi_{1,4 / 3}^{1,2}(M, N)$. Let $D$ be a domain in $M$. Choose local isothermal (i.e. conformal) coordinates $z=x_{1}+i x_{2}$ on $D$ and let $e_{1}=\partial_{x_{1}}, e_{2}=\partial_{x_{2}}$. We write

$$
\phi=\phi^{i} \partial y^{i}, \quad \psi=\psi^{j} \otimes \partial y^{j},
$$

and denote $\phi_{1}:=\nabla_{e_{1}} \phi=\phi_{x_{1}}, \phi_{2}:=\nabla_{e_{2}} \phi=\phi_{x_{2}}$. Then, we have

$$
\begin{gathered}
A\left(\phi_{\alpha}, \phi_{\alpha}\right)=\phi_{\alpha}^{i} \phi_{\alpha}^{j} \frac{\partial v_{l}^{i}}{\partial y^{j}} v_{l} \\
\mathcal{A}\left(\phi_{\alpha}, e_{\alpha} \cdot \psi\right)=\phi_{\alpha}^{i} e_{\alpha} \cdot \psi^{j} \otimes \frac{\partial v_{l}^{i}}{\partial y^{j}} v_{l} \\
\mathcal{P}\left(\mathcal{A}\left(\phi_{\alpha}, e_{\alpha} \cdot \psi\right) ; \psi\right)=\left(\frac{\partial v_{l}}{\partial y^{k}}\right)^{\top} \phi_{\alpha}^{i}\left\langle\psi^{k}, e_{\alpha} \cdot \psi^{j}\right\rangle \frac{\partial v_{l}^{i}}{\partial y^{j}} .
\end{gathered}
$$

Note that $\phi_{\alpha} \in T N$ and $\nu_{l} \in T^{\perp} N$, hence,

$$
\phi_{\alpha}^{i} v_{l}^{i}=0, \quad \forall \alpha, l
$$

It follows that

$$
A^{m}\left(\phi_{\alpha}, \phi_{\alpha}\right)=\phi_{\alpha}^{i} \phi_{\alpha}^{j} \frac{\partial v_{l}^{i}}{\partial y^{j}} v_{l}^{m}-\phi_{\alpha}^{i} \phi_{\alpha}^{j} \frac{\partial v_{l}^{m}}{\partial y^{j}} v_{l}^{i}=\phi_{\alpha}^{i}\left(\phi_{\alpha}^{j} \frac{\partial v_{l}^{i}}{\partial y^{j}} v_{l}^{m}-\phi_{\alpha}^{j} \frac{\partial v_{l}^{m}}{\partial y^{j}} v_{l}^{i}\right) .
$$

On the other hand, we have

$$
\mathcal{A}^{m}\left(\phi_{\alpha}, e_{\alpha} \cdot \psi\right)=\left(\phi_{\alpha}^{i} e_{\alpha} \cdot \psi^{j} \otimes \frac{\partial v_{l}^{i}}{\partial y^{j}} v_{l}\right)^{m}=\frac{\partial v_{l}^{i}}{\partial y^{j}} v_{l}^{m} \phi_{\alpha}^{i} e_{\alpha} \cdot \psi^{j}
$$

and

$$
\begin{aligned}
\operatorname{Re} \mathcal{P}^{m}\left(\mathcal{A}\left(\phi_{\alpha}, e_{\alpha} \cdot \psi\right) ; \psi\right) & =\operatorname{Re} \phi_{\alpha}^{i}\left\langle\psi^{k}, e_{\alpha} \cdot \psi^{j}\right\rangle \frac{\partial v_{l}^{i}}{\partial y^{j}}\left(\frac{\partial v_{l}}{\partial y^{k}}\right)^{\top, m} \\
& =\phi_{\alpha}^{i}\left(\left\langle\psi^{k}, e_{\alpha} \cdot \psi^{j}\right\rangle+\overline{\left\langle\psi^{k}, e_{\alpha} \cdot \psi^{j}\right\rangle}\right) \frac{\partial v_{l}^{i}}{\partial y^{j}}\left(\frac{\partial v_{l}}{\partial y^{k}}\right)^{\top, m} \\
& =\phi_{\alpha}^{i}\left(\left\langle\psi^{k}, e_{\alpha} \cdot \psi^{j}\right\rangle+\left\langle e_{\alpha} \cdot \psi^{j}, \psi^{k}\right\rangle\right) \frac{\partial v_{l}^{i}}{\partial y^{j}}\left(\frac{\partial v_{l}}{\partial y^{k}}\right)^{\top, m} \\
& =\phi_{\alpha}^{i}\left(\left\langle\psi^{k}, e_{\alpha} \cdot \psi^{j}\right\rangle-\left\langle\psi^{j}, e_{\alpha} \cdot \psi^{k}\right\rangle\right) \frac{\partial v_{l}^{i}}{\partial y^{j}}\left(\frac{\partial v_{l}}{\partial y^{k}}\right)^{\top, m} \\
& =\phi_{\alpha}^{i}\left\langle\psi^{k}, e_{\alpha} \cdot \psi^{j}\right\rangle\left(\frac{\partial v_{l}^{i}}{\partial y^{j}}\left(\frac{\partial v_{l}}{\partial y^{k}}\right)^{\top, m}-\frac{\partial v_{l}^{i}}{\partial y^{k}}\left(\frac{\partial v_{l}}{\partial y^{j}}\right)^{\top, m}\right)
\end{aligned}
$$

where $(\cdot)^{m}$ denotes the $m$-th component of a vector of $\mathbb{R}^{K}$. Thus, we can write (9) and (10) in the following extrinsic form in terms of the orthonomal frame field $v_{l}, l=d+1, \ldots, K$, for $T^{\perp} N$. 


$$
\begin{aligned}
-\Delta \phi^{m}= & \phi_{\alpha}^{i}\left(\phi_{\alpha}^{j} \frac{\partial v_{l}^{i}}{\partial y^{j}} v_{l}^{m}-\phi_{\alpha}^{j} \frac{\partial v_{l}^{m}}{\partial y^{j}} v_{l}^{i}\right) \\
& +\phi_{\alpha}^{i}\left\langle\psi^{k}, e_{\alpha} \cdot \psi^{j}\right\rangle\left(\frac{\partial v_{l}^{i}}{\partial y^{j}}\left(\frac{\partial v_{l}}{\partial y^{k}}\right)^{\top, m}-\frac{\partial v_{l}^{i}}{\partial y^{k}}\left(\frac{\partial v_{l}}{\partial y^{j}}\right)^{\top, m}\right), \\
\not \partial \psi^{m}= & \frac{\partial v_{l}^{i}}{\partial y^{j}} v_{l}^{m} \phi_{\alpha}^{i} e_{\alpha} \cdot \psi^{j} .
\end{aligned}
$$

\section{Regularity for weakly Dirac-harmonic maps}

When the target $N$ is the standard sphere $\mathbb{S}^{d} \subset \mathbb{R}^{d+1}$, Chen et al. [1] observed a Jacobian structure for Eq. (9), namely,

Proposition 3.1 Let $M$ be a Riemann surface with a fixed spin structure and $(\phi, \psi) \in$ $\chi_{1,4 / 3}^{1,2}\left(M, \mathbb{S}^{d}\right)$ a weakly Dirac-harmonic map from $M$ to $\mathbb{S}^{d}$. Let $D$ be a simply connected domain of $M$. Then there exists $\Omega=\left(\Omega^{i j}\right) \in W^{1,2}\left(D, \mathbb{R}^{(d+1) \times(d+1)}\right)$ such that

$$
-\Delta \phi=\frac{\partial \Omega}{\partial x} \frac{\partial \phi}{\partial y}-\frac{\partial \Omega}{\partial y} \frac{\partial \phi}{\partial x} .
$$

Moreover, they proved in [1] that

Theorem 3.1 Let $(\phi, \psi):\left(D, \delta_{\alpha \beta}\right) \rightarrow\left(N, g_{i j}\right)$ be a weakly Dirac-harmonic map. If $\phi$ is continuous, then $(\phi, \psi)$ is smooth.

Combining Proposition 3.1, Theorem 3.1 and Wente's lemma [11], the following regularity theorem was then proved in [1].

Theorem 3.2 Let $M$ be a Riemann surface with a fixed spin structure. Suppose that $(\phi, \psi) \in$ $\chi_{1,4 / 3}^{1,2}\left(M, \mathbb{S}^{d}\right)$ is a weakly Dirac-harmonic map from $M$ to $\mathbb{S}^{d}$. Then $\phi \in C^{0}$, and hence $(\phi, \psi)$ is smooth.

Before we consider the case of general target manifolds, let us recall Tristan Rivière's main result in [10].

Theorem 3.3 Let $K \in \mathbb{N}$, and let $D$ be the unit disk of $\mathbb{R}^{2}$. For every $\Omega=\left(\Omega_{j}^{i}\right)_{1 \leq i, j \leq K}$ in $L^{2}\left(D, \operatorname{so}(K) \otimes \mathbb{R}^{2}\right)$ (i.e. $\forall i, j \in 1, \ldots, K, \Omega_{j}^{i} \in L^{2}\left(D, \mathbb{R}^{2}\right)$ and $\left.\Omega_{j}^{i}=-\Omega_{i}^{j}\right)$, every $u \in W^{1,2}\left(D, \mathbb{R}^{K}\right)$ solving

$$
-\Delta u=\Omega \cdot \nabla u,
$$

is continuous, where the contracted notation in the above equation stands for $\forall i=1, \ldots, K$, $-\Delta u^{i}=\sum_{j=1}^{K} \Omega_{j}^{i} \cdot \nabla u^{j}$ in coordinates, where "." is the scalar product of two vectors in $\mathbb{R}^{2}$.

We observe now that when the target $N \subset \mathbb{R}^{d+1}$ is a compact hypersurface, (26) has a structure similar to (28).

Proposition 3.2 Let $M$ be a Riemann surface with a spin structure and $N \subset \mathbb{R}^{d+1}$ be a compact hypersurface. Let $(\phi, \psi) \in \chi_{1,4 / 3}^{1,2}(M, N)$ be a weakly Dirac-harmonic map from 
$M$ to $N$. Let $D$ be a domain in $M$ with local conformal coordinates $z=x_{1}+i x_{2}$. Then there exists $\Omega=\left(\Omega_{i}^{m}\right)_{1 \leq i, m \leq d+1}$ in $L^{2}\left(D, \operatorname{so}(d+1) \otimes \mathbb{R}^{2}\right)$ such that

$$
-\Delta \phi=\Omega \cdot \nabla \phi \text {. }
$$

Proof Let $N \subset \mathbb{R}^{d+1}$ be a compact hypersurface with normal $v$, then it follows from differentiating the equation $v \cdot v=1$ that $\nabla v \in T N$, hence

$$
\left(\frac{\partial v}{\partial y^{k}}\right)^{\top, m}=\left(\frac{\partial v}{\partial y^{k}}\right)^{m}=\left(\frac{\partial v^{m}}{\partial y^{k}}\right), \quad k, m=1,2, \ldots, d+1
$$

By (25), we get

$$
\begin{aligned}
\operatorname{Re} \mathcal{P}^{m}\left(\mathcal{A}\left(\phi_{\alpha}, e_{\alpha} \cdot \psi\right) ; \psi\right) & =\phi_{\alpha}^{i}\left\langle\psi^{k}, e_{\alpha} \cdot \psi^{j}\right\rangle\left(\frac{\partial v^{i}}{\partial y^{j}}\left(\frac{\partial v}{\partial y^{k}}\right)^{\top, m}-\frac{\partial v^{i}}{\partial y^{k}}\left(\frac{\partial v}{\partial y^{j}}\right)^{\top, m}\right) \\
& =\phi_{\alpha}^{i}\left\langle\psi^{k}, e_{\alpha} \cdot \psi^{j}\right\rangle\left(\frac{\partial v^{i}}{\partial y^{j}} \frac{\partial v^{m}}{\partial y^{k}}-\frac{\partial v^{i}}{\partial y^{k}} \frac{\partial v^{m}}{\partial y^{j}}\right) .
\end{aligned}
$$

On the other hand, from (23), we have

$$
A^{m}\left(\phi_{\alpha}, \phi_{\alpha}\right)=\phi_{\alpha}^{i}\left(\phi_{\alpha}^{j} \frac{\partial v^{i}}{\partial y^{j}} v^{m}-\phi_{\alpha}^{j} \frac{\partial v^{m}}{\partial y^{j}} v^{i}\right) .
$$

Denote

$$
\Omega_{i}^{m}:=\left(\begin{array}{c}
\lambda_{i}^{m} \\
\mu_{i}^{m}
\end{array}\right), \quad i, m=1,2, \ldots, d+1
$$

where

$$
\begin{aligned}
& \lambda_{i}^{m}:=\left(\frac{\partial v^{i}}{\partial y^{j}} v^{m}-\frac{\partial v^{m}}{\partial y^{j}} v^{i}\right) \phi_{1}^{j}+\left(\frac{\partial v^{i}}{\partial y^{j}} \frac{\partial v^{m}}{\partial y^{k}}-\frac{\partial v^{i}}{\partial y^{k}} \frac{\partial v^{m}}{\partial y^{j}}\right)\left\langle\psi^{k}, e_{1} \cdot \psi^{j}\right\rangle \\
& \mu_{i}^{m}:=\left(\frac{\partial v^{i}}{\partial y^{j}} v^{m}-\frac{\partial v^{m}}{\partial y^{j}} v^{i}\right) \phi_{2}^{j}+\left(\frac{\partial v^{i}}{\partial y^{j}} \frac{\partial v^{m}}{\partial y^{k}}-\frac{\partial v^{i}}{\partial y^{k}} \frac{\partial v^{m}}{\partial y^{j}}\right)\left\langle\psi^{k}, e_{2} \cdot \psi^{j}\right\rangle .
\end{aligned}
$$

Then, we can write (26) in the following form

$$
-\Delta \phi^{m}=\Omega_{i}^{m} \cdot \nabla \phi^{i}
$$

Now it remains to show that $\Omega=\left(\Omega_{i}^{m}\right)_{1 \leq i, m \leq d+1} \in L^{2}\left(D, \operatorname{so}(d+1) \otimes \mathbb{R}^{2}\right)$.

On the one hand, since $(\phi, \psi) \in \chi_{1,4 / 3}^{1,2}(M, N)$ is a weakly Dirac-harmonic map, we must have $\phi \in W^{1,2}$ and $\psi \in W^{1,4 / 3} \subset L^{4}$ (by the Sobolev embedding theorem). In view of (33), (34) and (35), we have

$$
\Omega_{i}^{m} \in L^{2}\left(D, \mathbb{R}^{2}\right), \quad \forall 1 \leq i, \quad m \leq d+1 .
$$

On the other hand, it is easy to see from (34) and (35) that both $\lambda_{i}^{m}$ and $\mu_{i}^{m}$ are real valued and are skew-symmetric with respect to the indices $i$ and $m$. Thus,

$$
\Omega=\left(\Omega_{i}^{m}\right)_{1 \leq i, m \leq d+1} \in L^{2}\left(D, \operatorname{so}(d+1) \otimes \mathbb{R}^{2}\right) .
$$

This completes the proof.

Proof of Theorem 1.1 The result follows from combining Proposition 3.2, Theorem 3.1 and Theorem 3.3. 
Acknowledgements The author would like to thank his advisor, Prof. Jürgen Jost, for his continued support and encouragement. He would also like to thank the referees for their valuable comments and suggestions.

\section{References}

1. Chen, Q., Jost, J., Li, J.Y., Wang, G.: Regularity theorems and energy identities for Dirac-harmonic maps. Math. Z. 251, 61-84 (2005)

2. Chen, Q., Jost, J., Li, J.Y., Wang, G.: Dirac-harmonic maps. Math. Z. 254, 409-432 (2006)

3. Deligne, P., et al. (eds.): Quantum Fields and Strings: A Course for Mathematicians. Vols. 1, 2, AMS \& Inst. Adv. Study (1999)

4. Friedrich, T.: Dirac operators in Riemannian geometry. Graduate Studies in Mathematics, vol. 25, xvi+195 pp. American Mathematical Society, Providence, RI (2000)

5. Hélein, F.: Régularité des applications faiblement harmoniques entre une surface et une varieté riemannienne. C. R. Acad. Sci. Paris Sér. I Math. 312, 591-596 (1991)

6. Hélein, F.: Harmonic Maps, Conservation Laws and Moving Frames, 2nd edn. Cambridge University Press, Cambridge (2002)

7. Hijazi O.: Spectral properties of the Dirac operator and geometrical structures. (English summary) Geometric methods for quantum field theory (Villa de Leyva, 1999), pp. 116-169, World Sci. Publ., River Edge, NJ. (2001)

8. Jost, J.: Riemannian geometry and geometric analysis. 4 edn. pp. xiv+566 Universitext SpringerVerlag, Berlin (2005)

9. Lawson, H.B., Michelsohn, M.-L.: Spin Geometry. Princeton University Press, Princeton. (1989)

10. Rivière, T.: Conservation laws for conformal invariant variational problems. Invent. Math. 168, 1-22 (2007)

11. Wente, H.: An existence theorem for surfaces of constant mean curvature. J. Math. Anal. Appl. 26, 318-344 (1969) 